\title{
Maximum-likelihood fitting of the 6dFGS peculiar velocities
}

\author{
Christina Magoulas, ${ }^{1,2}$ Christopher Springob, ${ }^{2}$ Matthew Colless, ${ }^{2}$ \\ D. Heath Jones, ${ }^{3}$ Lachlan Campbell, ${ }^{4}$ John Lucey, ${ }^{5}$ \\ and Jeremy Mould ${ }^{6}$ \\ ${ }^{1}$ University of Melbourne, Australia \\ email: c.magoulas@pgrad.unimelb.edu.au \\ ${ }^{2}$ Australian Astronomical Observatory, Australia \\ ${ }^{3}$ Monash University, Australia \\ ${ }^{4}$ University of Western Kentucky, USA \\ ${ }^{5}$ University of Durham, UK \\ ${ }^{6}$ Swinburne University, Australia
}

\begin{abstract}
We develop a robust Bayesian model to derive peculiar velocities and Fundamental Plane (FP) distances for a subsample of 9000 galaxies from the 6dF Galaxy Survey (6dFGS). These galaxies form the basis of 6dFGSv, the largest and most uniform galaxy peculiar-velocity sample to date. We perform a Bayesian analysis of the data set as a whole, determining cosmological parameters from the peculiar-velocity field (e.g., fitting $\beta$ and the bulk flow), by comparing to the field predicted from the redshift survey and assuming that the galaxy distribution traces the matter distribution.
\end{abstract}

Keywords. surveys, galaxies: distances and redshifts, cosmological parameters, large-scale structure of universe

\section{Introduction}

The $6 \mathrm{dFGS}$ peculiar-velocity survey (6dFGSv) comprises the brightest early-type galaxies in the primary 6dFGS redshift sample (Jones et al. 2009), out to $c z=16,500 \mathrm{~km} \mathrm{~s}^{-1}$. Distances and velocities of the 6dFGSv sample are measured using the Fundamental Plane (FP) relation, a tight correlation between the properties of early-type galaxies. The best-fitting FP and peculiar velocities have been determined for $~ 9000$ 6dFGS galaxies in near-infrared passbands (Magoulas et al. 2012).

\section{Bayesian Peculiar Velocity Distributions}

For the 6dFGSv sample we calculate a Bayesian probability distribution for the peculiar velocity of each galaxy, i.e. the probability density with velocity (nearly Gaussian in logarithmic units) given the observed angular radius, velocity dispersion, surface brightness and redshift. An example of the probability distribution of velocities for 10 galaxies in 6dFGSv is shown in Fig. 1. This Bayesian approach allows us to directly measure and interpret the posterior probability distribution of the peculiar velocities, rather than be limited to individual velocity measurements (C. Springob et al., in prep.). More specifically, we can use these individual velocity estimates to determine the cosmographic description of the 6dFGSv volume (see Section 3).

We compare the 6dFGSv peculiar-velocity field with the predicted model of Erdoğdu et al. (2012), derived from the final data release of the Two-Micron All Sky Redshift Survey (2MRs; Huchra et al. 2012), which is largely based on the 6dFGS primary redshift 


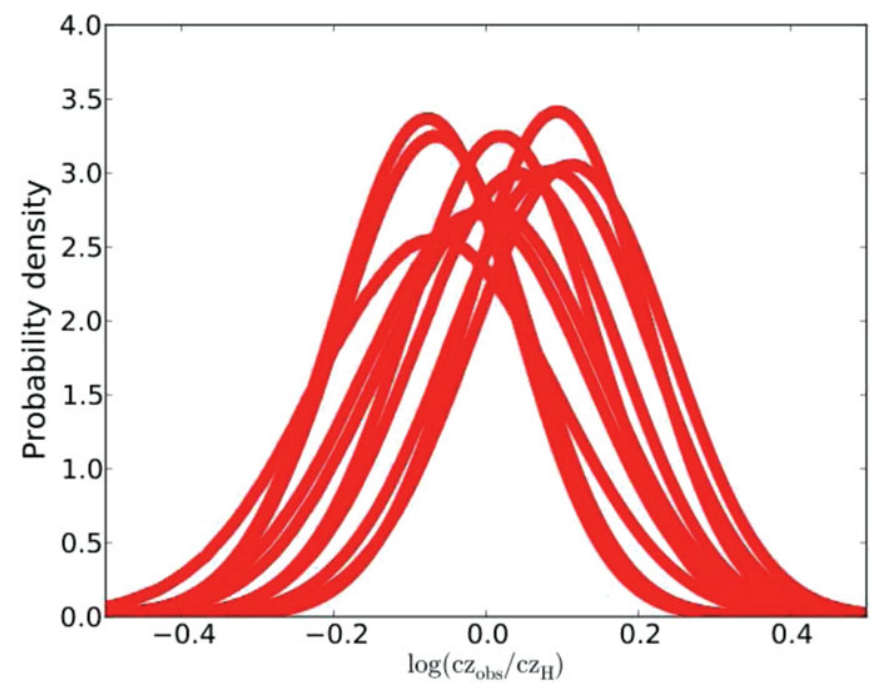

Figure 1. Posterior probability distribution of peculiar velocities for 10 typical galaxies in the 6dFGSv sample, which is (nearly) Gaussian in logarithmic units, as shown here, but is sampled evenly (and lognormally) in linear units.

sample. This model uses the methods of reconstruction outlined in Erdoğdu et al. (2006; where it is applied to a smaller sample) but has recently been updated for the deeper $K_{\mathrm{S}}=11.75$ mag-limited $2 \mathrm{MRS}$ by Erdoğdu et al. (2012). The extended 2MRs density and velocity fields are reconstructed using linear theory, assuming that the galaxy distribution in redshift space traces the mass distribution.

Additionaly, we can use Bayesian analysis of the data set as a whole for cosmological analysis (see Section 4) of the 6dFGS peculiar-velocity field, using a maximum-likelihood approach and incorporating a 3D Gaussian model to fit the FP (C. Springob et al., in prep.). We use this maximum-likelihood method to fit three types of peculiar-velocity models:

- The velocity field assuming that matter follows the galaxy distribution of $2 \mathrm{MRS}$, with $\beta$ as the free parameter.

- The velocity field assuming that matter follows the galaxy distribution of $2 \mathrm{MRS}$, with $\beta$ and a residual bulk flow as free parameters.

- The velocity field assuming that it can only be modelled by a total bulk flow.

\section{Cosmography from the 6dFGSv Velocity Field}

In Fig. 2 we show the Supergalactic cartesian coordinates ( $S G X$ versus $S G Y$ ) of the 6dFGSv galaxies in a slice though $S G Z$. We plot galaxy subsamples in two $S G Z$ slices, $-70<S G Z<-20 h^{-1} \mathrm{Mpc}$ (top) and $-20<S G Z<+20 h^{-1} \mathrm{Mpc}$ (bottom). The galaxies are coloured by their (smoothed) peculiar velocities measured from the 6dFGSv observations (right), which are in good agreement with those predicted from a reconstructed model of the velocity field (left). A dipole motion is evident in the lefthand panel of Fig. 2 as a prominence of positive peculiar velocities near the centre left and more negative velocities in the bottom right-hand corner. This dipole signal is larger than predicted and pointed towards the Shapley Supercluster. 

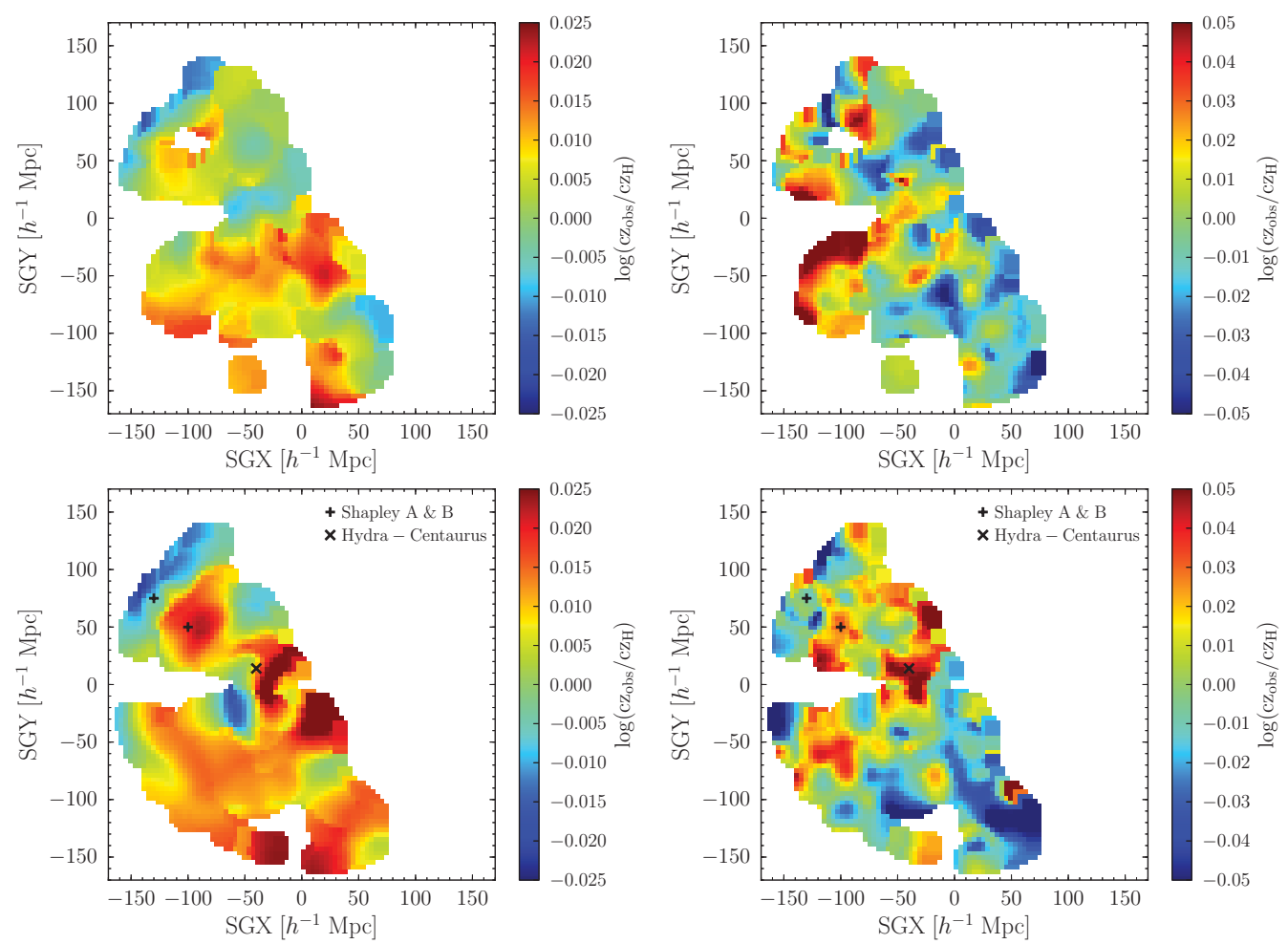

Figure 2. Large-scale velocity-field maps projected on the Supergalactic $S G X-S G Y$ plane with a pixel scale of $4 \times 4 h^{-1} \mathrm{Mpc}^{2}$. Each plot represents a subsample of galaxies divided by $S G Z$ into two slices: $-70<S G Z<-20 h^{-1} \mathrm{Mpc}$ (top) and $-20<S G Z<+20 h^{-1} \mathrm{Mpc}$ (bottom). Points are colour-coded by predicted peculiar velocities from the $2 \mathrm{MRS}$ density field (left) and observed peculiar velocities from 6dFGS (right), averaged using adaptive kernel smoothing. Some of the large structures in the 6dFGSv volume are also indicated, including the Shapley Supercluster and the Hydra-Centaurus Cluster.

\section{Cosmological results from the 6dFGSv Velocity Field}

Peculiar velocities distort the galaxy distribution in redshift space relative to the distribution in real space, thus affecting the determination of distance estimates. On large scales, the clustering of matter is amplified by the coherent infall of galaxies towards overdense regions. The effects of this type of distortion can be characterised by the linear redshift-space distortion parameter $\beta\left(=\Omega_{\mathrm{m}}^{0.55} / b\right)$, which links the total mass density and the bias in the distribution of galaxies relative to the underlying distribution of mass.

We can derive $\beta$ from a comparison of the matter and velocity fields from the observed peculiar velocities to those from the predictions of redshift surveys, in our case from 2MRs. Using the velocity-field model fitting to $\beta$ alone, we measure $\beta=0.29 \pm 0.06$, slightly lower than found from other studies. Discrepancies between $\beta$ values at this level may result from their sensitivity to the assumed value of linear bias between the mass and galaxy distributions, or from details such as the sample-selection limits, or differences in fitting methods. Also, the process of Wiener filtering used in the 2MRS model reconstruction smooths the density field in noisy regions (by construction) and, consequently, reduces the density contrast. Therefore, comparing the $6 \mathrm{dFGSv}$ velocities to a Wiener-smoothed reconstruction of the velocity field may lead to an underestimate of the value of $\beta$. This bias will need to be accounted for in any further analysis. 
Peculiar velocities are also used to study the coherent peculiar motion in a volume, or bulk flow, with respect to the cosmic microwave background rest frame. From the peculiar velocity field derived from the 6dFGSv, we have determined both the total bulk-flow motion and the residual bulk flow after subtracting the 2MRS-predicted velocity field. We recover a total bulk flow for $6 \mathrm{dFGSv}$ within $\sim 160 h^{-1} \mathrm{Mpc}$ of $337 \pm 66 \mathrm{~km} \mathrm{~s}^{-1}$ towards $(l, b)=\left(313^{\circ} \pm 9^{\circ}, 15^{\circ} \pm 10^{\circ}\right)$, suggesting coherent motion towards the Shapley Supercluster at $\left(312^{\circ}, 31^{\circ}\right)$. We also measure a residual bulk flow of $272 \pm 45 \mathrm{~km} \mathrm{~s}^{-1}$ in the direction of $\left(326^{\circ} \pm 13^{\circ}, 37^{\circ} \pm 14^{\circ}\right)$; a residual flow closer to the Shapley Supercluster in direction than the total bulk flow (Magoulas 2012).

After accounting for the fact that our survey volume is limited to the southern hemisphere, we find the 6dFGSv total bulk flow is not consistent with the predictions of $\Lambda C D M$ at greater than $2 \sigma$. The large amplitude of the residual dipole suggests that the bulk-flow motion of 6dFGSv is dominated by mass distributions unaccounted for by the 2 MRS volume or that the contribution of the Shapley Supercluster is underrepresented in the 2MRS reconstruction. However, we have yet to fully take into account the radial distribution of the sample and the variable errors in distance, which requires a more sophisticated radial weighting scheme, such as those used in Watkins et al. (2009) and Turnbull et al. (2012). The discrepancy between this large bulk flow and the expectation from $\Lambda \mathrm{CDM}$ may be explained by these effects, once they are corrected for.

\section{Summary}

In summary, 6dFGSv offers substantial improvement in the precision of bulk-flow measurements and cosmographic description of the nearby Universe. We have mapped the velocity field in the local Universe and compared it to the density field derived from redshift surveys. This leads to new constraints on the $\beta$ parameter linking mass density and galaxy bias as well as the cosmological bulk flow in the 6dFGSv volume. Here we present promising preliminary results from the 6dFGSv velocity field, with further detailed investigations to follow.

\section{References}

Erdoğdu, P., Lahav, O., Huchra, J. P., et al. 2006, MNRAS, 373, 45

Huchra, J. P., Macri, L. M., Masters, K. L., et al. 2012, ApJS, 199, 26

Jones, D. H., Read, M. A., Saunders, W., et al. 2009, MNRAS, 399, 683

Magoulas, C., Springob, C. M., Colless, M., et al. 2012, MNRAS, 427, 245

Magoulas, C. 2012b, Ph.D. Thesis, University of Melbourne, Australia

Turnbull, S., Hudson, M. J., Feldman, H. A., Hicken, M., Kirshner, R. P., \& Watkins, R. 2012, MNRAS, 420, 447

Watkins, R., Feldman, H. A., \& Hudson, M. J. 2009, MNRAS, 392, 743 